\title{
Analysis of chicken anemia virus genome: evidence of intersubtype recombination
}

Yassir M Eltahir ${ }^{1,3}$, Kun Qian $^{1}$, Wenjie Jin ${ }^{1}$ and Aijian Qin ${ }^{1,2^{*}}$

\begin{abstract}
Background: Chicken anemia virus (CAV) is the causative agent of chicken infectious anemia. CAV putative intergenotypic recombinants have been reported previously. This fact is based on the previous classification of CAV sequences into three genotypes. However, it is unknown whether intersubtype recombination occurs between the recently reported four CAV genotypes and five subtypes of genome sequences.

Results: Phylogenetic analysis, together with a variety of computational recombination detection algorithms, was used to investigate CAV approximately full genomes. Statistically significant evidence of intersubtype recombination was detected in the parent-like and two putative CAV recombinant sequences. This event was shown to occur between CAV subgroup A1 and A2 sequences in the phylogenetic trees.

Conclusions: We revealed that intersubtype recombination in CAV genome sequences played a role in generating genetic diversity within the natural population of CAV.
\end{abstract}

\section{Background}

Chicken anemia virus (CAV) was first reported in 1979 in specific-pathogen-free (SPF) chickens [1]. CAV belongs to the Circoviridae and is a non-enveloped, icosahedral virus with a negative-sense, single-stranded circular DNA. The viral genome consists of $2.3 \mathrm{~kb}$, with three partially overlapping open reading frames. CAV infection is an economically important clinical and subclinical disease in broiler chickens, with a worldwide distribution [2].

CAV isolates show extremely limited genetic variability worldwide [3]. All isolates of CAV are suspected to belong to a single serotype [4]. Little is known about CAV genome recombination analysis. CAV putative intergenotype recombinants have been reported to occurs in the virus gene VP1 and results in a new virus genotype [5].

Recently, after adding more CAV approximately full genome sequences to GenBank, CAV sequences arising from different parts of world have been categorized into four genotype groups (A-D) and five subtypes (A1, A2, A3, D1 and D2) [6]. Therefore, the necessity of exploring these CAV genotypes for evidence of recombination as an important tool for genetic variability has been raised,

\footnotetext{
* Correspondence: aijian@yzu.edu.cn

${ }^{1}$ Ministry of Education Key Lab for Avian Preventive Medicine, Yangzhou University, Yangzhou, 225009, PR China

Full list of author information is available at the end of the article
}

to establish if any would help in understanding the evolutionary process in the CAV genome.

Here, we report evidence of intersubtype recombination based on sequence analysis of the entire coding regions (VP1, VP2 and VP3) of CAV genomes.

\section{Methods \\ Samples}

DNA extraction, PCR screening, amplification of CAV genome, cloning and sequencing were carried out as previously described, briefly, primers VP1F: 5'AGCCGACCCCGAACCGCAAGAA'3 and VP1R: 5' TCA GGG CTG CGT CCC CCA GTA CA'3 were used to amplify the VP1 region and primers VP2F: 5' GCG CAC ATA CCG GTC GGC AGT'3 and VP2R: 5' GGG GTT CGG CAG CCT CAC ACT AT'3 were used to amplify the VP2 region [6] for 10 spleen samples collected between April 2010 and December 2010 at Yangzhou University Veterinary Hospital during necropsy. Samples originated from different commercial chicken farms in Anhui $(n=3)$ and Jiangsu $(n=7)$ provinces. For each samples, DNA extraction and PCR was run at least twice. Animal experiments were conducted in accordance with the guidelines provided by the Chinese Council on Animal Care. All experiments complied with institutional animal care guidelines
() Biomed Central

(C) 2011 Eltahir et al; licensee BioMed Central Ltd. This is an Open Access article distributed under the terms of the Creative Commons Attribution License (http://creativecommons.org/licenses/by/2.0), which permits unrestricted use, distribution, and reproduction in any medium, provided the original work is properly cited. 


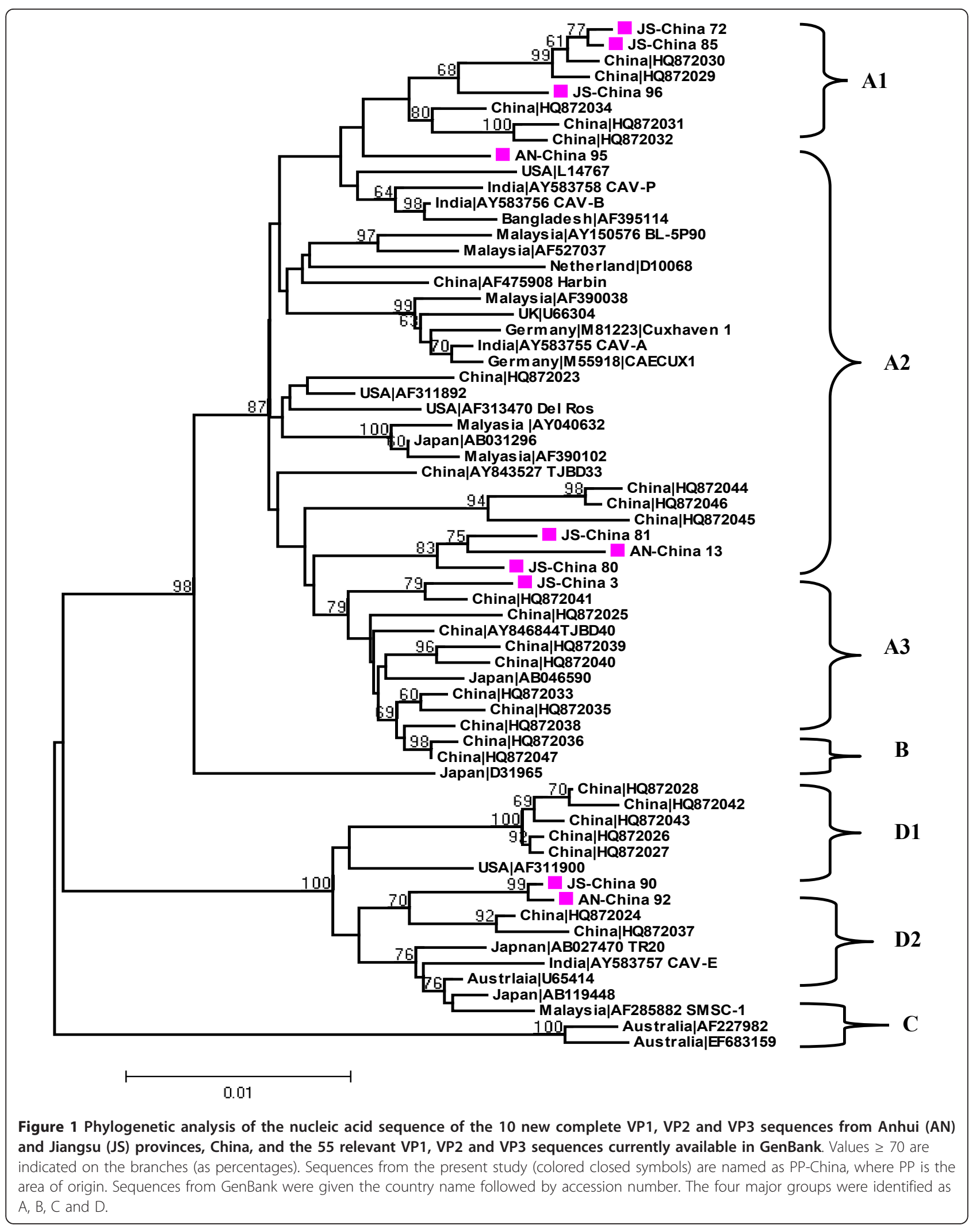


and were approved by University of Yangzhou Animal Care Committee (protocol number 06R015).

\section{Sequence analysis}

To look for recombination in CAV sequences, we used 55 published full genomes sequences that are currently available in GenBank, together with 10 sequences characterized in the present study. Multiple alignments of either CAV full or subgenomic regions were performed and analyzed using ClustalW [7]. Unrooted phylogenetic trees were constructed using the neighbor-joining (NJ) method, and visualized and edited using MEGA 3.1 software [8]. The evolutionary distances were estimated using the Kimura two-parameter method. Bootstrap analyses were performed with 1,000 repeat samples of the data sets. To reduce redundancy, isolates with previous reports of intergenotypic recombination [5] were excluded from analysis.

\section{Identification of recombination}

Recombination breakpoint events in the multiple alignments were detected with the Recombination Detection Program 3 (RDP3), using the automated suite of algorithms implemented in the RDP3 with default settings. These included RDP, GENECONV, BootScan, MaxChi, Chimaera, SiScan, Phyl- Pro, LARD, and 3Seq [9,10]. For the RDP algorithm, the reference sequence parameter (internal and external reference) was used as recommended by the manual. The multiple-aligned sequences were cut into sections at the positions where the putative recombination breakpoints were identified, and phylogenetic analyses were carried out by MEGA 3.1 software [8]. Confirmation of recombination break points was further carried out by bootscanning using SimPlot [11].

\section{Results}

\section{Sequences accession numbers}

CAV genome sequences characterized in this study were submitted to GenBank with accession numbers [GenBank: FR 850021, GenBank: FR 850022, GenBank: FR 850023, GenBank: FR 850024, GenBank: FR 850025, GenBank: FR 850026, GenBank: FR 850027, GenBank: FR 850028, GenBank: FR 850029, GenBank: FR 850030].

\section{Phylogenetic tree reconstructions}

When an NJ phylogenetic tree was reconstructed, we found that the high number of sequences included maintained the four genotype groups (A-D) and five subgroups (A1, A2, A3, D1 and D2) topology that was present in previously published trees (Figure 1) [6]. When the RDP, GENECONV, Boot Scan, MaxChi, Chimaera, SiScan, PhylPro, LARD, and 3Seq algorithms were used to detect recombination in CAV whole genome sequences, only one significant recombination event was detected. For this event, seven out of nine algorithms detected significant recombination at the same location in the CAV genome with $\mathrm{p}$ values ranging from $\left[6.215 \times 10^{-07}\right.$ to $\left.4.544 \times 10^{-2}\right]$ (Table 1). The location of two significant break points was in the VP1 coding region ( $n t$ positions 768 and 1286) of AN-China 13, which was considered as the daughter or recombinant, with the major parent being the HQ872045 and the minor parent being JS-China 72 .

\section{Confirmation of recombination event}

The region identified as containing the recombination breakpoint was confirmed by comparing the phylogenetic tree topologies of the entire CAV coding region without the recombinant region. The tree topology was essentially the same as for the whole genome. For the recombinant region, changes in tree topology and decreases in distance that separates the daughter (AN-China 13) and minor parent (JS-China 72) sequences were observed. AN-China 13 was located in subtype A2 in the tree constructed with the non-recombinant region (Figure 2), and was found within subtype A1 when the tree was developed with the recombinant region (Figure 3). Furthermore, when the recombinant minor parent and major parent sequences were further analyzed, recombination was supplemented and supported by identifying the two points of crossover by Boot Scan and Distance plot (Figure 4, 5). In addition, high similarity (99.3\%) between AN-China 13 and JSChina 72 was observed.

\section{Discussion}

Recombination is one of the evolutionary processes that shape the architecture of viral genomes. Ignoring the occurrence of recombination may influence the analysis of genetic data and the conclusions derived from it. Recombination analysis of CAV genomes is very limited. CAV intergenotype recombination break points in the VP1 region have been reported in only a single study using 35 CAV full genomes sequences [5]. However, to date, there is no evidence of inter- or intrasubtype recombination between CAV genomes, to the best of our knowledge. Therefore, the present study investigated CAV whole

Table 1 Recombination Statistics

\begin{tabular}{ccc}
\hline Algorithm & Recombination P-Value & nt Position \\
\hline RDP & $4.544 \times 10^{-02}$ & $768-1286$ \\
GENECONV & $n / a$ & $n / a$ \\
BootScan & $1.179 \times 10^{-02}$ & $768-1286$ \\
MAXcHl & $4.692 \times 10^{-03}$ & $768-1286$ \\
Chimaera & $1.768 \times 10^{-04}$ & $768-1286$ \\
SiScan & $6.215 \times 10^{-07}$ & $768-1286$ \\
PhylPro & $n / a$ & $n / a$ \\
LARD & $1.499 \times 10^{-05}$ & $768-1286$ \\
3Seq & $1.612 \times 10^{-05}$ & $768-1286$ \\
\hline
\end{tabular}



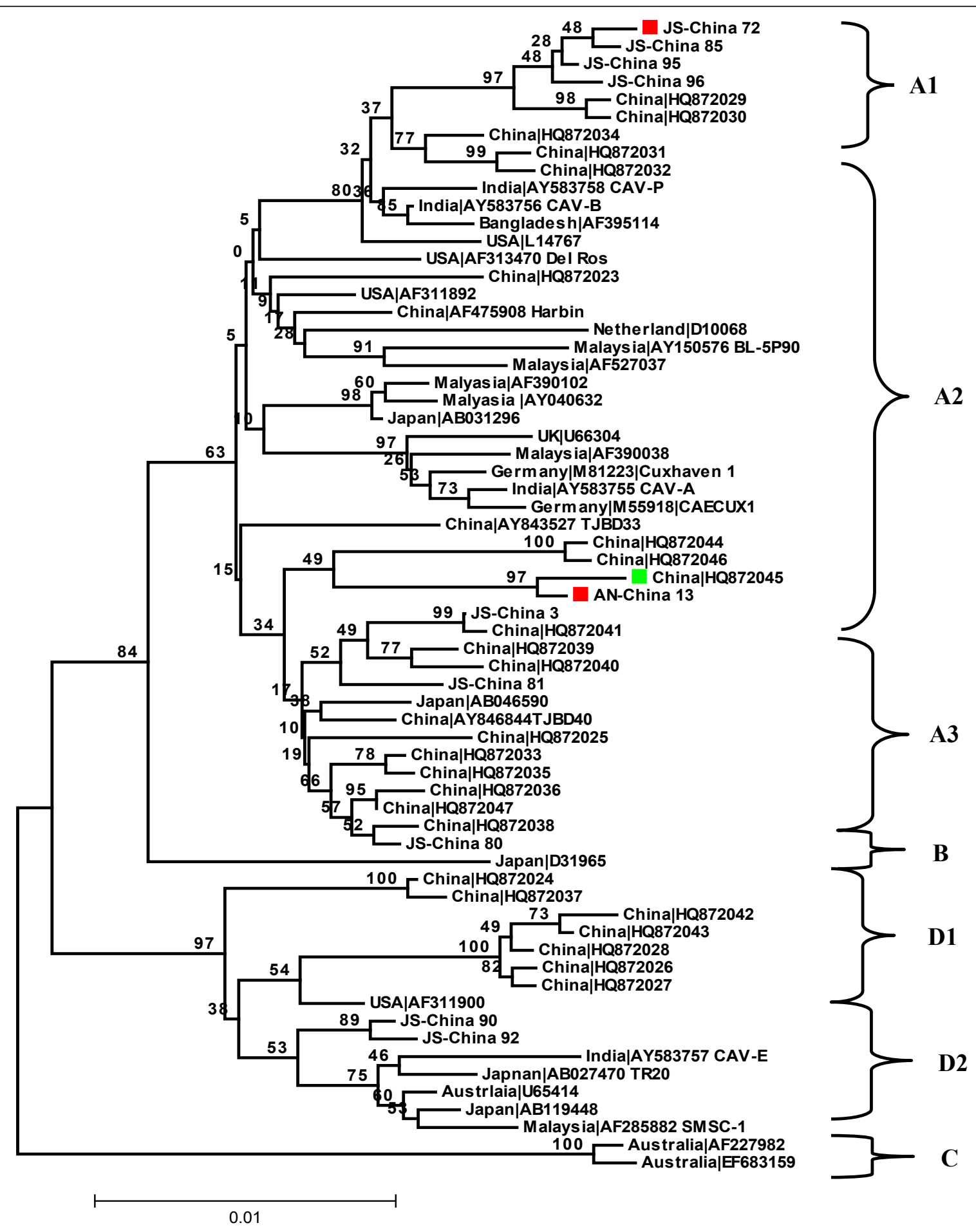

Figure 2 Phylogenetic analysis of the non-recombinant region of the 10 new CAV sequences from Anhui (AN) and Jiangsu (JS) provinces, China, and the $\mathbf{5 5}$ relevant CAV sequences currently available in GenBank. Sequences from the present study are named as PP-China, where PP is the area of origin. Red and green colored closed symbols represent recombinant, minor parent and major parent sequences respectively. GenBank sequences were given the country name followed by accession number. The four major groups were identified as $A, B, C$ and D. 


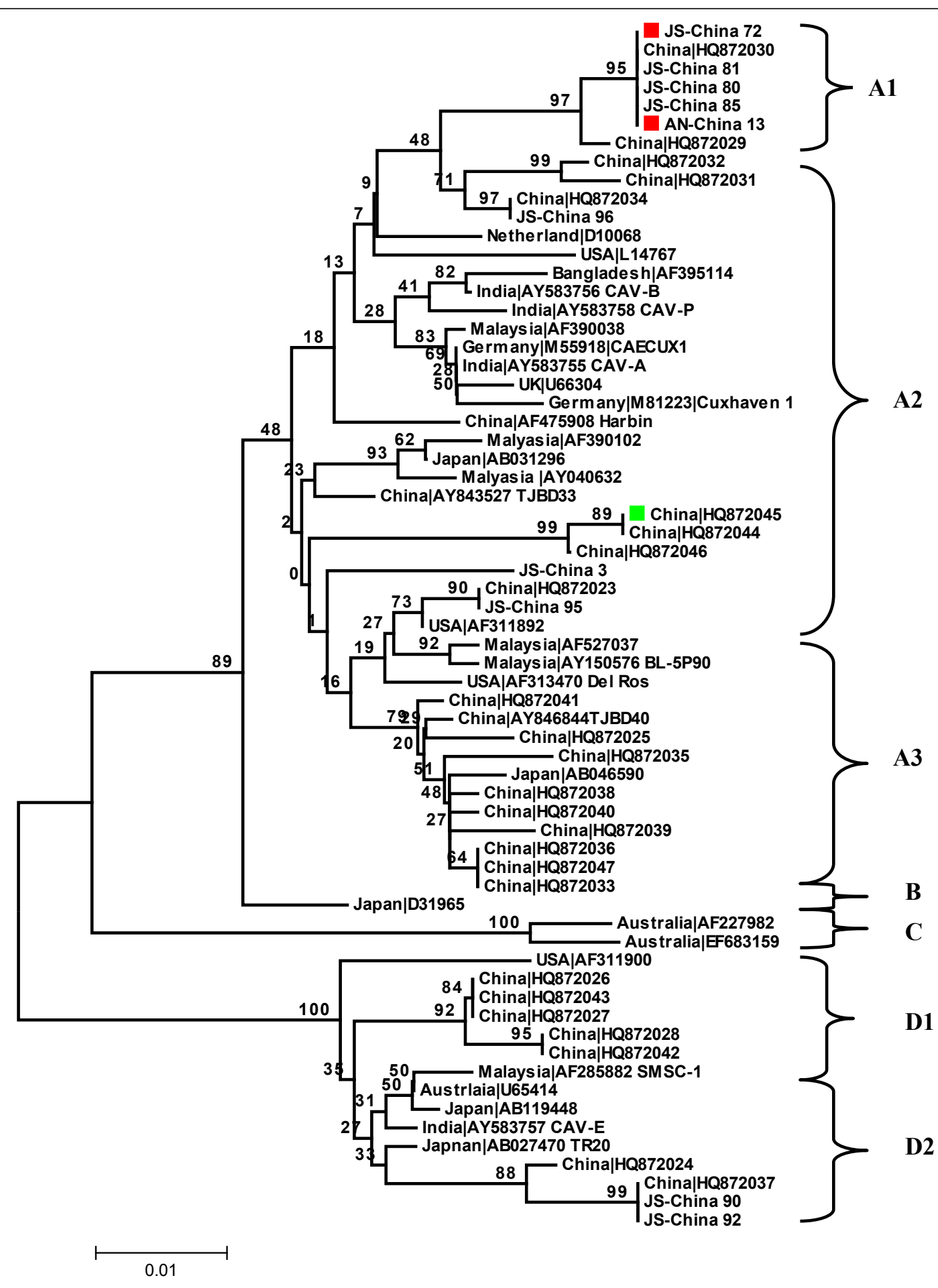

Figure 3 Phylogenetic analysis of the recombinant region of the 10 new CAV sequences from Anhui (AN) and Jiangsu (JS) provinces, China, and the 55 relevant CAV sequences currently available in GenBank. Sequences from the present study are named as PP-China, where PP is the area of origin. Red and green colored closed symbols represent recombinant, minor parent and major parent sequences respectively. GenBank sequences were given the country name followed by accession number. The four major groups were identified as A, B, C and $\mathrm{D}$. 


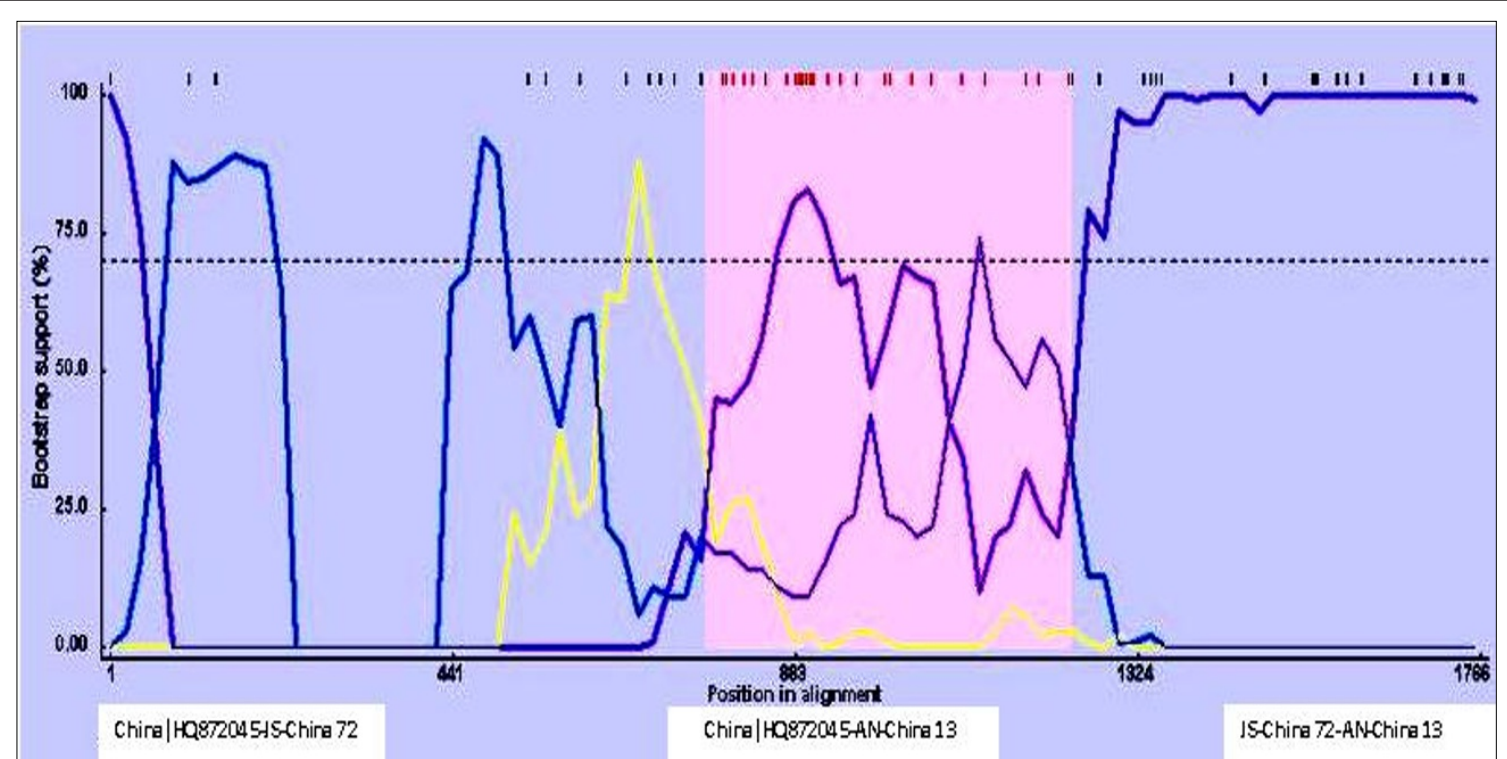

Figure 4 Bootscan analysis of the recombinant, major parent and the minor parent sequences. Bootscan was based on pairwise distance model with the window size 200, step size 50, and 1000 bootstrap replicates generated by the RDP3 program.

genome sequences to explore the effect of recombination in the genetic variation of CAV sequences.

Here, we report the first evidence of intersubtype recombination events in CAV genome sequences amplified from two different areas (Anhui and Jiangsu provinces) in China. Intersubtype mosaic viruses are the results of co-infection events in geographic regions where multiple subtypes circulate $[12,13]$. Similarly, co-infection with CAV that has been documented by sequencing electropherogram double peaks and cloning has also been reported [14]. Moreover, intersubtype recombination in other virus subtypes has also been reported $[15,16]$.

Natural recombination in the VP1 of the CAV genome, with a resultant new genotype, has suggested speeding up of CAV evolution [5]. However, it should be noted that these findings were based on a limited number of CAV

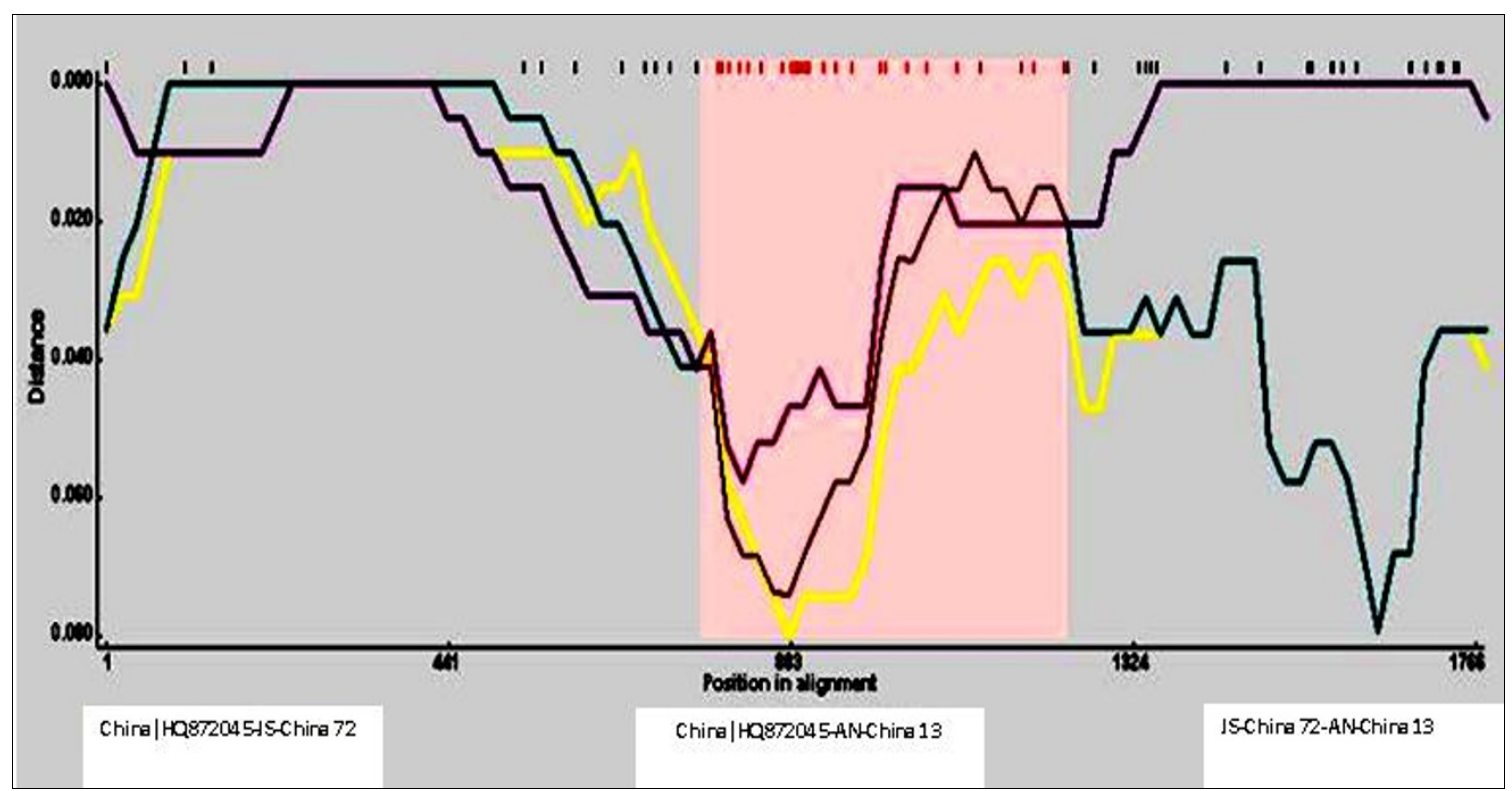

Figure 5 Distance plot analysis of the recombinant, major parent and the minor parent sequences. Pair-wise distances for distance plot were calculated for each window using DNADIST. 
sequences and the previous categorization of CAV sequences into three genotypes. In contrast, despite adding more sequences here, we confirmed that CAV genomes can be categorized into their recent four genotype groups (A-D) and five subtypes (A1, A2, A3, D1 and D2) [6].

The recombination breakpoints detected in CAV subgroup $\mathrm{A} 1$ and $\mathrm{A} 2$ sequences in the present study were also found in the VP1 protein. This resulted in the sequence from subgroup A2 being located within subgroup A1. It is known that most nucleotide variation in the CAV genome is observed in VP1, in addition to the fact that virulence of CAV is also determined by VP1 $[17,18]$. The cumulative evidence of recombination breakpoints in the VP1 of the CAV genome may be indicative of favorable selection for recombination in this variable region. Sequence variations at specific sites between HIV-1 isolates have been reported to introduce unique recombination hotspots and increase recombination frequencies in the C3/V4 env region [19]. However, whether there is correlation between the highest genetic variability in CAV genomes observed in the VP1 and recombination still needs to be investigated.

\section{Conclusions}

Using bioinformatics analysis, we were able to detect only a single incidence of CAV intersubtype recombination that contributed to CAV genetic variation. More detailed analysis for further CAV intragenotypic recombination is still required.

\section{Acknowledgements \\ The research was supported by the Program for Changjiang Scholars and Innovative Research Team in University (IRT0978) and the Major Basic Research of Natural Science Foundation of the Jiangsu Higher Education Institutions of China (Grant No. 07KJA23021).}

\section{Author details}

${ }^{1}$ Ministry of Education Key Lab for Avian Preventive Medicine, Yangzhou University, Yangzhou, 225009, PR China. ${ }^{2}$ Key Laboratory of Jiangsu Preventive Veterinary Medicine, Yangzhou University, 225009, PR China. ${ }^{3}$ Department of Preventive Medicine and Veterinary Public Health, Faculty of Veterinary Science, University of Nyala, Nyala, Sudan.

\section{Authors' contributions}

YME designed the study, carried out the experiments, analyzed the data, and drafted the manuscript. QA supervised all the experiments and participated in the data analysis. JW and QK discussed and prepared the final report. All authors have read and approved the final manuscript.

\section{Competing interests}

The authors declare that they have no competing interests.

Received: 20 April 2011 Accepted: 10 November 2011 Published: 10 November 2011

\section{References}

1. Yuasa N, Taniguchi T, Yoshida I: Isolation and some characteristics of an agent inducing anemia in chicks. Avian Dis 1979, 23:366-385.
2. Todd D, Creelan JL, Mackie DP, Rixon F, McNulty MS: Purification and biochemical characterization of chicken anemia agent. J Gen Virol 1990, 71:819-823.

3. Van Santen VL, Li L, Hoerr FJ, Lauerman LH: Genetic characterization of chicken anemia virus from commercial broiler chickens in Alabama. Avian Dis 2001, 45(2):373-388

4. Koch G, van Roozelaar DJ, Verschueren CA, van der Eb AJ, Noteborn MH: Immunogenic and protective properties of chicken anaemia virus proteins expressed by baculovirus. Vaccine 1995, 13(8):763-770.

5. He CQ, Ding NZ, Fan W, Wu YH, Li JP, Li YL: Identification of chicken anemia virus putative intergenotype recombinants. Virology 2007, 15(366 (1)):1-7.

6. Eltahir YM, Qian K, Jin W, Wang P, Qin A: Molecular epidemiology of chicken anemia virus in commercial farms in China. Virol J 2011, 145.

7. Thompson JD, Gibson TJ, Plewniak F, Jeanmougin F, Higgins DG: The CLUSTAL_X windows interface: flexible strategies for multiple sequence alignment aided by quality analysis tools. Nucleic Acids Res 1997, 25:(24), 4876-4882.

8. Kumar S, Tamura K, Nei M: MEGA3: Integrated software for Molecular Evolutionary Genetics Analysis and sequence alignment. Brief Bioinform 2004, 5:150-163.

9. Martin DP, Williamson C, Posada D: RDP2: recombination detection and analysis from sequence alignments. Bioinformatics 2005, 21:260-262.

10. Martin DP, Posada D, Crandall KA, Williamson C: A modified bootscan algorithm for automated identification of recombinant sequences and recombination breakpoints. AIDS Res Hum Retroviruses 2005, 21:98-102.

11. Lole KS, Bollinger RC, Paranjape RS, Gadkari D, Kulkarni SS, Novak NG, Ingersoll R, Sheppard HW, Ray SC: Full-length human immunodeficiency virus type 1 genomes from subtype C-infected seroconverters in India, with evidence of intersubtype recombination. J Virol 1999, 73:152-160.

12. Salminen $\mathrm{MO}$, Carr JK, Robertson DL: Evolution and probable transmission of intersubtype recombinant human immunodeficiency virus type 1 in a Zambian couple. J Virol 1997, 71:2647-2655

13. Gao F, Robertson DL, Carruthers CD: A comprehensive panel of near-fulllength clones and reference sequences for nonsubtype $B$ isolates of human immunodeficiency virus type 1. J Virol 1998, 72:5680-5698.

14. Ducatez MF, Owoade AA, Abiola JO, Muller CP: Molecular epidemiology of chicken anemia virus in Nigeria. Arch Virol 2006, 151:97-111.

15. Fomsgaard A, Vinner L, Therrien D, Jørgensen LB, Nielsen C, Mathiesen L, Pedersen C, Corbet S: Full-length characterization of A1/D intersubtype recombinant genomes from a therapy-induced HIV type 1 controller during acute infection and his noncontrolling partner. AIDS Res Hum Retroviruses 2008, 24(3):463-72.

16. Kurbanov F, Tanaka Y, Fujiwara K, Sugauchi F, Mbanya D, Zekeng L, Ndembi N, Ngansop C, Kaptue L, Miura T, Ido E, Hayami M, Ichimura $H_{\text {, }}$ Mizokami M: A new subtype (subgenotype) Ac (A3) of hepatitis B virus and recombination between genotypes $A$ and $E$ in Cameroon. J Gen Virol 2005, 86:2047-56.

17. Todd D, Scott AN, Ball NW, Borghmans BJ, Adair BM: Molecular basis of the attenuation exhibited by molecularly cloned highly passaged chicken anemia virus isolates. J Virol 2002, 76:8472-8474.

18. Yamaguchi S, Kaji N, Mase M, Tsukamoto K, Tanimura N, Yuasa N: Identification of a genetic determinant of pathogenicity in chicken anaemia virus. J Gen Virol 2001, 82:1233-1238.

19. Baird HA, Gao Y, Galetto R, Lalonde M, Anthony RM, Giacomoni V, Abreha M, Destefano JJ, Negroni M, Arts EJ: Influence of sequence identity and unique breakpoints on the frequency of intersubtype HIV-1 recombination. Retrovirology 2006, 12:3-91.

doi:10.1186/1743-422X-8-512

Cite this article as: Eltahir et al:: Analysis of chicken anemia virus genome: evidence of intersubtype recombination. Virology Journal 2011 8:512. 